\title{
Healthy ageing, resilience and wellbeing
}

\author{
T. D. Cosco ${ }^{1,2 *}$, K. Howse ${ }^{1}$ and C. Brayne ${ }^{3}$ \\ ${ }^{1}$ Oxford Institute of Population Ageing, University of Oxford, Oxford, UK \\ ${ }^{2}$ MRC Unit for Lifelong Health and Ageing, London, UK \\ ${ }^{3}$ Cambridge Institute of Public Health, University of Cambridge, Cambridge, UK
}

The extension of life does not appear to be slowing, representing a great achievement for mankind as well as a challenge for ageing populations. As we move towards an increasingly older population we will need to find novel ways for individuals to make the best of the challenges they face, as the likelihood of encountering some form of adversity increases with age. Resilience theories share a common idea that individuals who manage to navigate adversity and maintain high levels of functioning demonstrate resilience. Traditional models of healthy ageing suggest that having a high level of functioning across a number of domains is a requirement. The addition of adversity to the healthy ageing model via resilience makes this concept much more accessible and more amenable to the ageing population. Through asset-based approaches, such as the invoking of individual, social and environmental resources, it is hoped that greater resilience can be fostered at a population level. Interventions aimed at fostering greater resilience may take many forms; however, there is great potential to increase social and environmental resources through public policy interventions. The wellbeing of the individual must be the focus of these efforts; quality of life is an integral component to the enjoyment of additional years and should not be overlooked. Therefore, it will become increasingly important to use resilience as a public health concept and to intervene through policy to foster greater resilience by increasing resources available to older people. Fostering wellbeing in the face of increasing adversity has significant implications for ageing individuals and society as a whole.

First published online 6 July 2017

Key words: Healthy ageing, mental health, resilience, wellbeing.

\section{Introduction}

As a result of increasing longevity and decreasing fertility, unprecedented numbers of individuals are reaching older ages. Alongside this extension of the quantity of life there are myriad implications for the quality of that life. The process of ageing is profoundly heterogeneous, with great variations in the lived experiences of individuals' physical, social and psychological functioning domains across the life course. Striving for high levels of functioning across these many domains of life is a central goal of healthy ageing. Models of 'Successful Aging' are varied, but centre on the avoidance of negative aspects of ageing, e.g., morbidity, and the fostering of positive aspects, e.g., active engagement (Cosco et al. 2014a). These models do not, however, actively include components of adversity (Rowe \& Kahn, 1987, 1997), a component actively incorporated in models of resilience.

Stemming from developmental psychology (Rutter, 1987, 1993), theories of resilience have been integrated into models of healthy ageing (Pruchno et al. 2015). Resilience theories share a common idea that individuals who manage to navigate adversity and maintain high

* Address for correspondence: T. D. Cosco, Oxford Institute of Population Ageing, University of Oxford, Oxford, UK.

(Email: theodore.cosco@ageing.ox.ac.uk) levels of functioning demonstrate resilience. This framework aligns closely with the 'wellbeing paradox' (Windle, 2011, 2012), which demonstrates that as individuals grow older they may experience higher levels of wellbeing than in previous decades. The public health implications of fostering greater levels of wellbeing in individuals who experience adversity are diverse and significant, ranging from decreased caregiver burden to increased workplace productivity. As the population grows older, facing age-related adversities at unprecedented rates, the ability of individuals to demonstrate resilience, i.e., high levels of wellbeing, is becoming increasingly important for older adults, caregivers and clinicians.

\section{Healthy ageing}

Up until the 1960s, the prevailing theories of the ageing process considered older age a progressive, linear decline towards death (Cumming, 1968). Of particular note was Cumming \& Henry's Disengagement theory, which identified successful ageing as the ability to detach oneself from the activities of mid-life as a kind of preparation for death (1968). These theories were poorly received amongst older adults due to their negative focus. In response to these more negative theories of ageing, Robert Havighurst developed the Activity 
Theory (1961), which - in contrast to Cumming and Henry's Disengagement Theory - identified successful ageing as staying actively involved in the activities enjoyed in earlier life. This positive perspective on the process of ageing catalysed an entire field of research into healthy or 'successful' ageing.

Following Havighurst's (1961) 'Successful Aging', a letter was published in Science by John W. Rowe and Robert Kahn 'Human Aging: usual and successful'. This article propelled the focus of successful ageing into the mainstream media and popularised the idea amongst researchers and clinicians. In this and subsequent models, Rowe and Kahn suggested that the process of ageing well included three components: (i) the avoidance of disease or illness, (ii) maintenance of physical and cognitive function and (iii) active engagement. The combination of a high level of each of these components was suggested to constitute successful ageing.

The Rowe \& Kahn model of successful ageing has received considerable criticism, refinement and expansion over the years. In a recent review of operational definitions of successful ageing, Cosco et al. (2014a) revealed more than 100 unique operationalisations. A subsequent systematic review by the same authors revealed that these perspectives of successful ageing from researchers did not align with those of older adults themselves (Cosco et al. 2013, 2014b). Laypersons suggest that a focus on psychosocial components - or more subjective components - are integral to healthy ageing whilst researchers focused more on biomedical - or more objective - components. These conceptualisations of successful ageing are useful research tools, e.g., allowing predictors of the highest levels of functioning to be identified, as well as individual- and populationlevel goals towards which to strive. However, by definition, a large proportion of the ageing populace cannot achieve these goals due to the presence of age-related disease and declines in functioning.

\section{Resilience}

The term 'resilience' originates from the Latin 'resiliens', which refers to an elastic or pliant quality of a substance (Joseph, 1994). This ability of a substance to be able to 'bounce back' has subsequently been applied to individuals. However, since the inception of the term, there have been many conceptualisations and operationalisations of resilience (Cosco et al. 2016). At the core of this framework is some form of adversity and a positive response to this adversity.

\section{Origins}

The field of human resilience has its origins in developmental psychology, where researchers examined the trajectories of 'high-risk' populations of children. Researchers sought to identify how certain children born into adverse situations managed to avoid psychopathologies. This first wave of resilience research sought to differentiate between resilient and nonresilient individuals (Reich et al. 2010). Subsequent waves of research expanded these definitions to understand the processes of resilience, to test these processes through intervention, and finally to integrate other fields into psychological and psychiatric frameworks of resilience (Reich et al. 2010). In addition to these developments in the ways that the resilience concept has been developed and approached, the temporal span of resilience has widened from children, to mid-life, to older adults (Ong et al. 2009). Despite the popularity of resilience research in ageing, there was a notable time lag in the inclusion of older adults into this conceptual framework. During this lag period, a large proportion of investment in research was directed at biomedical or pharmaceutical solutions for age-related functional declines, rather than invoking individual, social or environmental resources.

\section{Healthy ageing and resilience}

Traditional models of healthy ageing suggest that having a high level of functioning across a number of domains is a requirement; resilience is present when a high level of functioning is present after a negative event, or some form of adversity. Consequently, these two fit well together: an individual that maintains a high level of functioning in the absence of adversity is healthily ageing, whilst one who maintains the same high functioning levels despite having experienced adversity is both resilient and healthily ageing. This addition of adversity to the healthy ageing model via resilience makes this concept much more accessible and more amenable to the ageing population.

The greatest predictor of functional disability is age (Manton et al. 1997); older adults are highly likely to experience adversity in some form during the ageing process, be it the decline of physical or cognitive faculties, the loss of a loved one, or the diminishing of social networks. Given the prevalence of these adversities, putting forward a model that suggests that individuals must maintain a high level of functioning across all of these domains excludes a significant portion of the population, if not almost all, by certain ages. Particularly in binary conceptualisations of healthy ageing, older adults are much less likely to be considered as 'healthy ageing' as they age. Using a binary conceptualisation of successful ageing in the Cambridge City over-75 Cohort, Cosco et al. (2014) 
identified $<1 \%$ of the sample population of individuals aged 75 and above as successfully ageing across seven waves of data collection. Clearly, the presence of some form of adversity makes binary conceptualisations of healthy ageing prohibitively exclusive, limiting the usefulness of healthy ageing as a public health tool.

The responses individuals have to these adversities are directly related to his/her resilience. Therefore, this is a more useful construct insofar as individuals who face adversity or experience functional declines can still demonstrate resilience, whereas they would not be classified as healthily ageing. There is an increasing trend towards the use of a resilience framework in older adults given that more individuals can be included in this model (Pruchno et al. 2015). Further, the identification of protective factors for resilience might be generalisable to a greater number of individuals as they face adversity across the life course.

Healthy ageing and resilience concepts fit well within each other; individuals can foster both resilience and healthy ageing simultaneously. Resilience is, however, inclusive of an adversity component, making it a much more widely applicable model amongst older adults. As Fig. 1 demonstrates, individuals that maintain a consistently high level of functioning, regardless of adversity encountered, demonstrate healthy ageing whilst individuals that exhibit greater levels of functioning than would be expected given their level of adversity experienced demonstrate resilience.

\section{Reserve hypotheses}

Theories of reserve suggest that individuals accrue resources over time to combat challenges in life. In the same manner that an individual that puts a portion of his/her paycheck into a savings account is better able to handle unexpected expenses, e.g., vehicle breakdown, than individuals that have not put money away, the accrual of resources is a protective factor for individuals in a number of domains. The cognitive reserve hypothesis has received a great deal of attention with respect to staving off cognitive decline. In this model, it is suggested that individuals who engage in activities that build up their cognitive faculties, for example through advanced education (Stern, 2007, 2012), can accommodate physiological insults, e.g., plaques and tangles, which are often present in individuals with dementia (Brayne et al. 2010). Of the suggested causal pathways for this cognitive reserve is the invoking of compensatory mechanisms that are better able to circumvent cognitive deficits, allowing the individuals to have fewer clinical presentations of cognitive decline than an individual with the same physiological deficits, but do not have the reserve facilities to compensate (Brayne et al. 2010). Individuals are able to accommodate increasing levels of adversity through the invoking of reserves; however, once these reserves have been exhausted the individual may experience clinical manifestations.

Given the multidimensional nature of healthy ageing and resilience, there are many domains in which individuals can build reserve. In the lifecourse model of multimorbidity resilience it is suggested that there are three areas that work in concert to foster resilience in the face of adversity: individual, social and environmental resources (Wister et al. 2016). Through the invoking of these different areas, individuals can build reserves to foster greater resilience and healthy ageing.

\section{Adding life to years}

There have been large increases in the length of life; however, the quality of that life has been comparatively under studied in contemporary medical literature. What we do with these extended years of life is of immense importance. Theorists have suggested a number of ways in which quality of life and wellbeing can be promoted in order to address the changing needs of ageing populations and older individuals. The compression of morbidity hypothesis proposes the objective to decrease the years of illness relative to the life span, the so-called 'squaring' of one's functioning trajectory (Fries, 1989; Fries et al. 2011).

Ageing is associated with declines in a host of functional domains, often leading to multimorbidity and frailty. Consequently individuals may experience a long slow decline towards death. Compression of morbidity suggests that this trajectory remains on a high plateau before a steep decline before death. By compressing the amount of time an individual spends facing functional deficits to an abrupt terminal decline towards death, greater wellbeing can be experienced for a larger proportion of his/her life span.

Wellbeing is an integral component of adding life to years in the presence of increasing longevity. As the wellbeing paradox highlights, individuals often experience high levels of wellbeing despite age-related declines (Windle, 2011). This trend of greater wellbeing and resilience in the face of adversity is one of significant public health interest and importance. If we are able to foster greater wellbeing for older adults, despite these increasing challenges, there is potential to decrease the burden on caregivers, clinicians and on ageing individuals themselves.

Greater wellbeing has been associated with reduced monetary burden and increased economic contributions. Previous studies have demonstrated greater employment outcomes in individuals with greater 


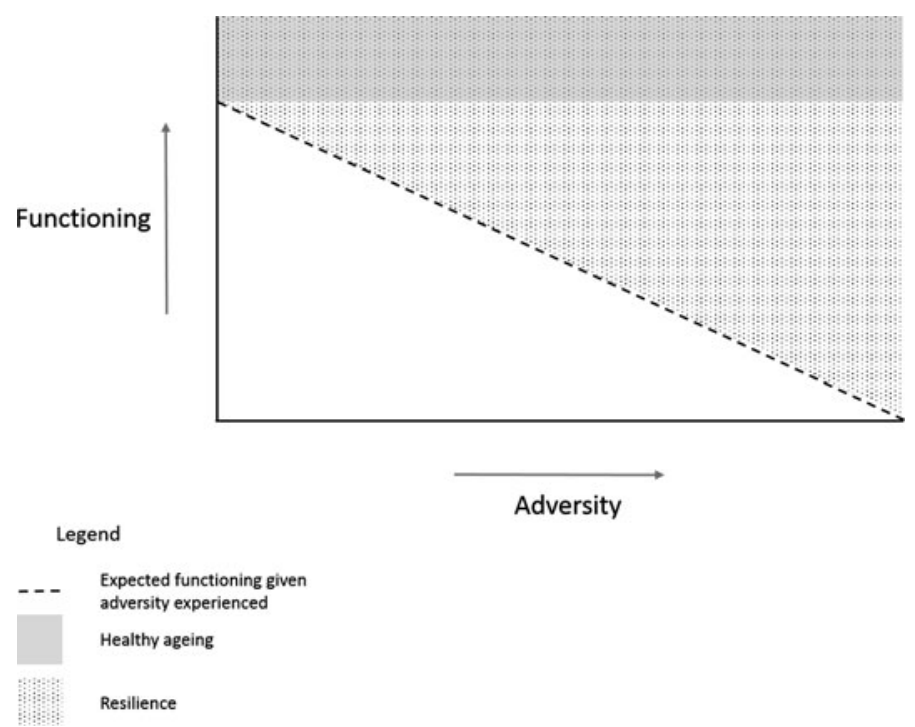

Fig. 1. The relationship between healthy ageing and resilience with regards to functioning and adversity.

levels of wellbeing report lower levels of healthcare costs, fewer unscheduled employment absences and greater productivity (Sears et al. 2013). These findings are also present in individuals with chronic disease, notably fibromyalgia, chronic low back pain and ankylosing spondylitis. Boonen et al. (2005) identified a significant relationship between lower wellbeing and increased overall costs across these three chronic conditions, suggesting that increasing wellbeing in adversity has significant monetary implications. Through the fostering of greater wellbeing in adversity individuals can continue to contribute to the economy and simultaneously reduce healthcare costs and caregiver burden. This knowledge provides a two-pronged approach to addressing this issue.

\section{Greater need for resilience}

With increasing longevity there will inevitably be increasing adversity for older adults. As we continue to push the boundaries of human lifespan the likelihood of individuals experiencing greater levels of adversity increases proportionally. Consequently, we will need to find solutions at a population levels that can address these needs and promote wellbeing. Resilience is a multifaceted construct with relevance to many domains, e.g., physical and psychological, making it a valuable public health objective. In contrast to models of successful ageing that have the avoidance of decline as an objective, resilience actively includes these adversities, making it a much more accessible and valuable public health tool. Through public policy initiatives that promote resilience at a population level, it is hoped that better wellbeing will be experienced by more of the burgeoning population of older adults.

Through increasing reserves and protective resources, it is hoped that resilience in older age can be increased at a population level. As highlighted by the lifecourse model of multimorbidity (Wister et al. 2016), engagement of individual, social and environmental resources may enable older adults to face the challenges of ageing head on and to foster greater wellbeing in their own lives. These types of asset approaches to resilience emphasise the contributions of resources beyond the individual, e.g., social and environmental, that have strong potential for policy intervention. For example, the World Health Organization has developed 'Global Age-friendly Cities: A Guide' (World Health Organization, 2007), documenting the ways in which cities can optimise opportunities for older adults and, in turn, increase their individual, social and environmental resources. Adopting these types of policies has the potential to have significant implications for wellbeing. Through greater social capital and better environmental fit and mastery through policy, it is hoped that large-scale population-level improvements in resilience can be made and the many benefits of greater wellbeing can be reaped.

\section{Future directions}

The extension of life does not appear to be slowing, representing a great achievement for mankind as well as a challenge for ageing populations. As we move towards an increasingly older population we will need to find novel ways for individuals to make 
the best of the challenges they face, as the likelihood of encountering some form of adversity increases with age. Through asset-based approaches, such as the invoking of individual, social and environmental resources, it is hoped that greater resilience can be fostered at a population level. Interventions aimed at fostering greater resilience may take many forms; however, there is great potential to increase social and environmental resources through the public policy interventions. The wellbeing of the individual must be the focus of these efforts; quality of life is an integral component to the enjoyment of additional years and should not be overlooked. Therefore, it will become increasingly important to use resilience as a public health concept and to intervene through policy to foster greater resilience by increasing resources available to older people. Fostering wellbeing in the face of the increasing adversity has significant implications for ageing individuals and society as a whole.

\section{Financial Support}

This research received no specific grant from any funding agency, commercial or not-for-profit sectors.

\section{Conflict of Interest}

None.

\section{References}

Boonen A, Van Den Heuvel R, Van Tubergen A, Goossens M, Severens JL, Van Der Heijde D, Van Der Linden S (2005). Large differences in cost of illness and wellbeing between patients with fibromyalgia, chronic low back pain, or ankylosing spondylitis. Annals of the Rheumatic Diseases 64, 396-402.

Brayne C, Ince PG, Keage HA, McKeith IG, Matthews FE, Polvikoski T, Sulkava R (2010). Education, the brain and dementia: neuroprotection or compensation? Brain 133, 2210-2216.

Cosco TD, Kaushal A, Hardy R, Richards M, Kuh D, Stafford M (2016). Operationalising resilience in longitudinal studies: a systematic review of methodological approaches. Journal of Epidemiology and Community Health 77, 98-104.

Cosco TD, Prina AM, Perales J, Stephan B, Brayne C (2013). Lay perspectives of successful ageing: a systematic review and meta-ethnography. BMJ Open 3, e002710.

Cosco TD, Prina AM, Perales J, Stephan B, Brayne C (2014a). Operational definitions of successful aging: a systematic review. International Psychogeriatrics 26, 373-381.
Cosco TD, Prina AM, Perales J, Stephan B, Brayne C (2014b). Whose 'successful ageing'? Lay- and researcher-driven conceptualisations of ageing well. European Journal of Psychiatry 28, 124-130.

Cosco TD, Stephan B, Brayne C \& The CC75C (2014). (Unsuccessful) binary modelling of successful aging in the oldest old: a call for continuum-based measures. Journal of the American Geriatrics Society 62, 1597-1598.

Cumming E (1968). New thoughts on the theory of disengagement. International Journal of Psychiatry 6, 53-67.

Fries J (1989). The compression of morbidity: near or far? Milbank Quarterly 67, 208-232.

Fries JF, Bruce B, Chakravarty E (2011). Compression of morbidity 1980-2011: a focused review of paradigms and progress. Journal of Aging Research 2011, 1-10.

Havighurst R (1961). Successful aging. Gerontologist 1, 8-13.

Joseph J (1994). The Resilient Child: Preparing Today's Youth for Tomorrow's World. Perseus: USA.

Manton KG, Corder L, Stallard E (1997). Chronic disability trends in elderly United States populations: 1982-1994. Proceedings of the National Academy of Sciences of the United States of America 94, 2593-2598.

Ong AD, Bergeman CS, Boker SM (2009). Resilience comes of age: defining features in later adulthood. Journal of Personality 77, 1777-1804.

Pruchno R, Heid AR, Genderson MW (2015). Resilience and successful aging: aligning complementary constructs using a life course approach. Psychological Inquiry 26, 200-207.

Reich JW, Zautra AJ, Hall S (2010). Handbook of Adult Resilience. The Guildford Press: New York, NY.

Rowe J, Kahn R (1987). Human aging: usual and successful. Science 237, 143-149.

Rowe J, Kahn R (1997). Successful aging. Gerontologist 37, 433-440.

Rutter M (1987). Psychosocial resilience and protective mechanisms. American Journal of Orthopsychiatry 57, 316-331.

Rutter M (1993). Resilience: some conceptual considerations. Journal of Adolescent Health 14, 626-631, 690-696.

Sears LE, Shi Y, Coberley CR, Pope JE (2013). Overall well-being as a predictor of health care, productivity, and retention outcomes in a large employer. Population Health Management 16, 397-405.

Stern Y (2007). Cognitive Reserve: Theory and Applications. Taylor \& Francis: Philadelphia, PA.

Stern Y (2012). Cognitive reserve in ageing and Alzheimer's disease. Lancet Neurology 11, 1006-1012.

Windle G (2011). What is resilience? A review and concept analysis. Reviews in Clinical Gerontology 21, 152-169.

Windle G (2012). The contribution of resilience to healthy ageing. Perspectives on Public Health 132, 159-160.

Wister AV, Coatta KL, Schuurman N, Lear SA, Rosin M, MacKey D (2016). A lifecourse model of multimorbidity resilience: theoretical and research developments. International Journal on Aging and Human Development 82, 290-313.

World Health Organization (2007). Global Age-friendly Cities: a Guide. WHO Press: Geneva. 